\title{
Stability Problems for Chua System with One Linear Control
}

\author{
Camelia Pop Arieşanu \\ "Politehnica" University of Timişoara, Department of Mathematics, 2 Victoriei Place, 300006 Timişoara, Romania \\ Correspondence should be addressed to Camelia Pop Arieşanu; cariesanu@yahoo.com
}

Received 16 January 2013; Revised 11 March 2013; Accepted 13 March 2013

Academic Editor: Chein-Shan Liu

Copyright (C) 2013 Camelia Pop Arieșanu. This is an open access article distributed under the Creative Commons Attribution License, which permits unrestricted use, distribution, and reproduction in any medium, provided the original work is properly cited.

A Hamilton-Poisson realization and some stability problems for a dynamical system arisen from Chua system are presented. The stability and dynamics of a linearized smooth version of the Chua system are analyzed using the Hamilton-Poisson formalism. This geometrical approach allows to deduce the nonlinear stabilization near different equilibria.

\section{Introduction}

The Chua system was proposed by Chua as a model of a nonlinear electrical circuit for the generation of chaotic oscillations. For some parameter values it has chaotic behavior. In this work we apply the geometric methods underlying the Hamilton-Poisson approach to analyze the characteristic features of the system. This geometrical approach makes it possible to find new properties that facilitate the dynamics description of the system, as well as its stability analysis. The work is divided as follows: the first part presents a short overview of the Chua's system and its Hamilton-Poisson realization. The Casimir function can be found only for a specific relation between the system's parameters. Finding the Casimir for the general case remains an open problem. The stability problem is discussed in the last section. Applying a control about $O y$ axis we stabilized the only one equilibrium state of our dynamics. The nonlinear stability of the equilibrium state is studied via energy-Casimir method. For a specific case of the system's parameters, the equilibrium point admits periodic orbits, presented in the last paragraph, too.

\section{The Poisson Geometry Associated to a Smooth Linear Version of Chua System}

The original Chua system of differential equations on $R^{3}$ has the following form (see [1] for details):

$$
\begin{gathered}
\dot{x}=\alpha(y-h(x)), \\
\dot{y}=x-y+z,
\end{gathered}
$$

$$
\dot{z}=-\beta y,
$$

where the characteristic function $h$ is a piecewise linear function, with $\alpha, \beta$ being real parameters. First of all let us take as a characteristic function in system (1) the function $h(x)=0$ so the system (1) becomes

$$
\begin{gathered}
\dot{x}=\alpha y, \\
\dot{y}=x-y+z, \\
\dot{z}=-\beta y .
\end{gathered}
$$

The goal of this section is to try to find a HamiltonPoisson structure for the system (2). In order to do this, let us recall very briefly the definitions of general Poisson manifolds and the Hamilton-Poisson systems.

Definition 1 (see [2,3]). Let $M$ be a smooth manifold and let $C^{\infty}(M)$ denote the set of the smooth real functions on $M$. A Poisson bracket on $M$ is a bilinear map from $C^{\infty}(M) \times C^{\infty}(M)$ into $C^{\infty}(M)$, denoted as

$$
(F, G) \longmapsto\{F, G\} \in C^{\infty}(M), \quad F, G \in C^{\infty}(M)
$$

which verifies the following properties:

(i) skew-symmetry:

$$
\{F, G\}=-\{G, F\}
$$


(ii) jacobi identity:

$$
\{F,\{G, H\}\}+\{G,\{H, F\}\}+\{H,\{F, G\}\}=0 ;
$$

(iii) leibniz rule:

$$
\{F, G \cdot H\}=\{F, G\} \cdot H+G \cdot\{F, H\}
$$

Proposition 2 (see $[2,3])$. Let $\{\cdot, \cdot\}$ be a Poisson structure on $R^{n}$. Then for any $f, g \in C^{\infty}\left(R^{n}, R\right)$ the following relation holds:

$$
\{f, g\}=\sum_{i, j=1}^{n}\left\{x_{i}, x_{j}\right\} \frac{\partial f}{\partial x_{i}} \frac{\partial g}{\partial x_{j}} .
$$

Let the matrix given by

$$
\Pi=\left[\left\{x_{i}, x_{j}\right\}\right] .
$$

Proposition 3 (see $[2,3]$ ). Any Poisson structure $\{\cdot, \cdot\}$ on $R^{n}$ is completely determined by the matrix $\Pi$ via the relation:

$$
\{f, g\}=(\nabla f)^{t} \Pi(\nabla g) \text {. }
$$

Definition 4 (see $[2,3])$. A Hamilton-Poisson system on $R^{n}$ is the triple $\left(R^{n},\{\cdot, \cdot\}, H\right)$, where $\{\cdot, \cdot\}$ is a Poisson bracket on
$R^{n}$ and $H \in C^{\infty}\left(R^{n}, R\right)$ is the energy (Hamiltonian). Its dynamics is described by the following differential equations system:

$$
\dot{x}=\Pi \cdot \nabla H,
$$

where $x=\left(x_{1}, x_{2}, \ldots, x_{n}\right)^{t}$.

Definition 5 (see $[2,3]$ ). Let $\{\cdot, \cdot\}$ be a Poisson structure on $R^{n}$. A Casimir of the configuration $\left(R^{n},\{\cdot, \cdot\}\right)$ is a smooth function $C \in C^{\infty}\left(R^{n}, R\right)$ which satisfies

$$
\{f, C\}=0, \quad \forall f \in C^{\infty}\left(R^{n}, R\right) .
$$

Now, following the above results, we are able to prove the following.

Proposition 6. The system (2) has the Hamilton-Poisson realization:

$$
\left(R^{3}, \Pi:=\left[\Pi^{i j}\right], H\right)
$$

where

$$
\Pi=\left[\begin{array}{ccc}
0 & \frac{1}{\alpha-\beta}(x-y+z) & y \\
-\frac{1}{\alpha-\beta}(x-y+z) & 0 & \frac{1}{\alpha-\beta}(x-y+z) \\
-y & -\frac{1}{\alpha-\beta}(x-y+z) & 0
\end{array}\right],
$$

The next step is to try to find the Casimir functions of the configuration described by Proposition 1. Since the Poisson structure is degenerate, we can try to obtain Casimir functions. The defining equation for the Casimir functions, denoted by $C$, is $\Pi^{i j} \partial_{j} C=0$. The determination of a Casimir in a finite dimensional Hamilton-Poisson system could be done via the algebraic method of Hernández-Bermejo and Fairén (see [4]).

Let us observe that the rank of $\Pi$ is constant and equal to 2 . Then, there exists only one functionally independent Casimir associated to our structure. Following the method described in [4], the Casimir function is the solution of

$$
d z=-d x-\frac{y(\beta-\alpha)}{x-y+z} d y
$$

For the integrability of this Pfaifian system, one needs an integrant factor to transform it into an equivalent one such that the above form is exact. The existence of such an integrant factor is guaranteed by the Frobenius theorem. If $\beta-\alpha=-2$, an integrant factor is $\varphi(x, y, z):=x+y+z$ and one obtains a Casimir of our configuration given by the following expression:

$$
\begin{aligned}
C(x, y, z)= & \frac{1}{3} x^{3}-\frac{2}{3} y^{3} \\
& +\frac{1}{3} z^{3}+x^{2} z-x y^{2}+x z^{2}-y^{2} z
\end{aligned}
$$

Consequently we have derived the following result.

Proposition 7. If $\beta-\alpha=-2$ then the real smooth function $C: R^{3} \rightarrow R$,

$$
\begin{aligned}
C(x, y, z)= & \frac{1}{3} x^{3}-\frac{2}{3} y^{3} \\
& +\frac{1}{3} z^{3}+x^{2} z-x y^{2}+x z^{2}-y^{2} z
\end{aligned}
$$

is the only one functionally independent Casimir of the Hamilton-Poisson realization of the system (2). 


\section{Stability and Stabilization by One Linear Control}

Let us pass now to discuss the stability problem (see [3] for details) of the system (2). It is not hard to see that the equilibrium states of our dynamics are

$$
e^{M}=(M, 0,-M), \quad M \in R
$$

Let $A$ be the matrix of the linear part of our system. The characteristic roots of $A\left(e^{M}\right)$ are given by

$$
\lambda_{1}=0, \quad \lambda_{2,3}=\frac{1}{2}(-1 \pm \sqrt{1+4(\alpha-\beta)}) .
$$

If $\alpha \leq \beta$ then the equilibrium states $e^{M}, M \in R$, are spectrally stable. Moreover, the equilibrium states $e^{M}, M \in R$, are unstable.
Let us consider now the case $\alpha \leq \beta$. We shall prove that the equilibrium states

$$
e^{M}=(M, 0,-M), \quad M \in R,
$$

of the system (2) may be nonlinear stabilized by a particular linear control applied to the axis $\mathrm{Oy}$.

The system (2) with one control about the axis $O y$ can be written in the following form:

$$
\begin{gathered}
\dot{x}=\alpha y, \\
\dot{y}=x-y+z+u, \\
\dot{z}=-\beta y,
\end{gathered}
$$

where $u \in C^{\infty}\left(R^{3}, R\right)$. In all that follows we shall employ the feedback:

$$
u=k y, \quad k \in R
$$

Proposition 8. The controlled system (20)-(21) is a HamiltonPoisson mechanical system with the phase space $P=R^{3}$, the Poisson structure

$$
\Pi:=\left[\begin{array}{ccc}
0 & \frac{1}{\alpha-\beta}(x+(k-1) y+z) & y \\
-\frac{1}{\alpha-\beta}(x+(k-1) y+z) & 0 & \frac{1}{\alpha-\beta}(x+(k-1) y+z) \\
-y & -\frac{1}{\alpha-\beta}(x+(k-1) y+z) & 0
\end{array}\right],
$$

and the Hamiltonian $H$ given by

$$
H=\beta x+\alpha z,
$$

where $\alpha, \beta \in R, \alpha \neq \beta$.

Remark 9. If $k=1$ then the function $C \in C^{\infty}\left(R^{3}, R\right)$ given by

$$
C(x, y, z)=x^{2}+2 x z-(\alpha-\beta) y^{2}+z^{2}
$$

is a Casimir of the configuration from Proposition 8.

If $k \neq 1$ finding the Casimir of the structure remains an open problem.

The phase curves of the dynamics (20)-(21) are the intersections of the surfaces: $H=$ const. and $C=$ const. see Figure 1.

Proposition 10. If $k=1$ then the system (20)-(21) may be realized as a Hamilton-Poisson system in an infinite number of different ways; that is, there exists infinitely many different (in general nonisomorphic) Poisson structures on $R^{3}$ such that the system (20)-(21) is induced by an appropriate Hamiltonian.

Proof. The triples:

$$
\left(R^{3}\{\cdot, \cdot\}_{a b}, H_{c d}\right)
$$

where

$$
\begin{gathered}
\{f, g\}_{a b}=\nabla C \cdot(\nabla f \times \nabla g), \quad \forall f, g \in C^{\infty}\left(R^{3}, R\right), \\
C_{a b}=a C+b H, \quad H_{c d}=c C+d H, \\
a, b, c, d \in R, a d-b c=1, \\
H=\frac{1}{\alpha-\beta}(\beta x+\alpha z), \\
C=\frac{1}{2}\left(x^{2}-(\alpha-\beta) y^{2}+2 x z+z^{2}\right),
\end{gathered}
$$

define Hamilton-Poisson realizations of the dynamics (20)(21).

Using now the energy-Casimir method we can prove the following.

Proposition 11. If $\alpha<\beta$ then the controlled system (20)-(21) may be nonlinear stabilized about the equilibrium states $e^{M}=$ $(M, 0,-M), M \in R$ for $k=1$.

Proof. Let

$$
H_{\varphi}=C+\varphi(H)=x^{2}+2 x z-(\alpha-\beta) y^{2}+z^{2}+\varphi(\beta x+\alpha z)
$$




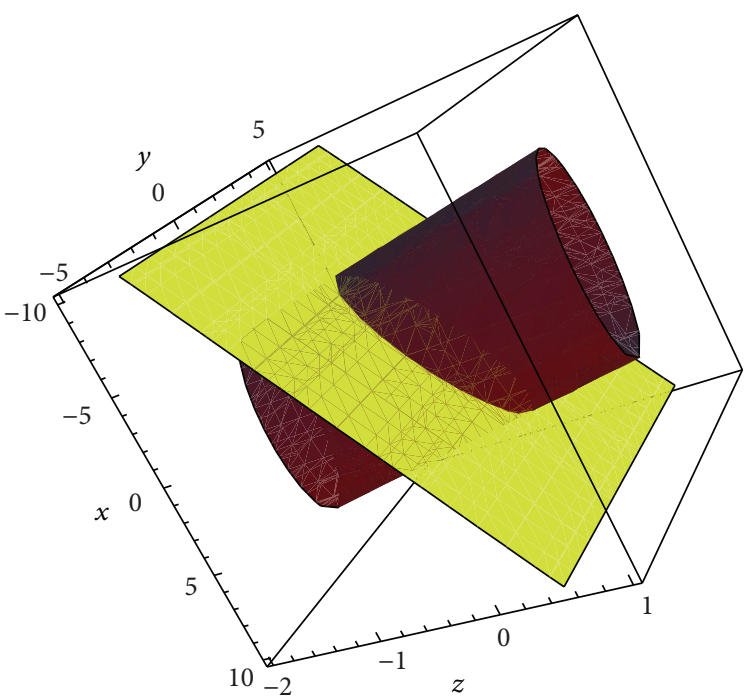

(a)

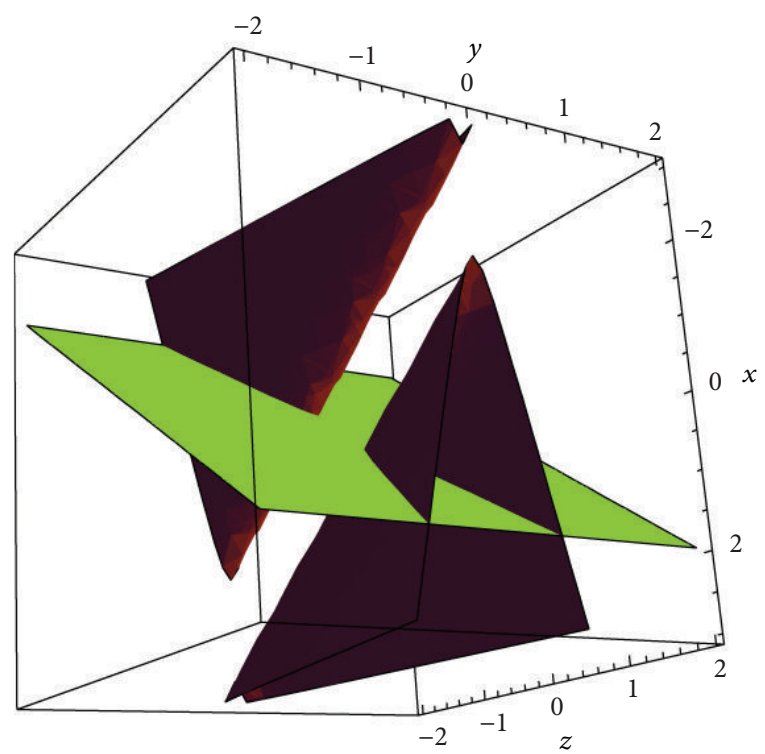

(b)

FIgURE 1: The phase curves of the system (20)-(21) for $a=-20, b=2$ and $a=20, b=-20$, respectively.

be the energy-Casimir function, where $\varphi: R \rightarrow R$ is a smooth real valued function defined on $R$.

Now, the first variation of $H_{\varphi}$ is given by

$$
\begin{aligned}
\delta H_{\varphi}= & 2(x+z) \delta x-2(\alpha-\beta) y \delta y \\
& +2(x+z) \delta z+\dot{\varphi}(\beta x+\alpha z)(\beta \delta x+\alpha \delta z) .
\end{aligned}
$$

This equals zero at $e^{M}$ if and only if

$$
\dot{\varphi}(\beta M-\alpha M)=0 .
$$

The second variation of $H_{\varphi}$ is given by

$$
\begin{aligned}
\delta^{2} H_{\varphi}= & 2(\delta x)^{2}-2(\alpha-\beta)(\delta y)^{2} \\
& +2(\delta z)^{2}+4 \delta x \delta z+\ddot{\varphi} \cdot(\beta \delta x+\alpha \delta z)^{2} .
\end{aligned}
$$

If $\alpha<\beta$ and having chosen $\varphi$ such that

$$
\begin{aligned}
& \dot{\varphi}(\beta M-\alpha M)=0, \\
& \ddot{\varphi}(\beta M-\alpha M)>0,
\end{aligned}
$$

we can conclude that the second variation of $H_{\varphi}\left(e^{M}\right)$ is positively defined and thus $e^{M}$ is nonlinearly stable.

Proposition 12. If

$$
\beta>0, \quad \alpha<0, \quad \beta=|\alpha|,
$$

then near to $e^{M}=(M, 0,-M), M \in R^{*}$, the reduced dynamics has, for each sufficiently small value of the reduced energy, at least 1 periodic solution whose period is close to $\pi / \sqrt{-2 \alpha}$.
Proof. We will use Moser's theorem for zero eigenvalue; see [5] for details.

(i) The restriction of our dynamics (20)-(21) to the coadjoint orbit:

$$
x-z=2 M
$$

gives rise to a classical Hamiltonian system.

(ii) Consider $\operatorname{span}_{R}\left(\nabla H\left(e^{M}\right)\right)=V_{\lambda=0}=\operatorname{span}_{R}\left(\left[\begin{array}{c}1 \\ 0 \\ -1\end{array}\right]\right)$, where

$$
V_{\lambda=0}=\left\{\left[\begin{array}{l}
x \\
y \\
z
\end{array}\right] \in R^{3} \mid A\left(e^{M}\right)\left[\begin{array}{l}
x \\
y \\
z
\end{array}\right]=\left[\begin{array}{l}
0 \\
0 \\
0
\end{array}\right]\right\},
$$

with $A\left(e^{M}\right)$ being the matrix of the linear part of the system (20)-(21) at the equilibrium of interest $e^{M}$.

(iii) The matrix of the linear part of our reduced dynamics to (20)-(21) has purely imaginary roots at the equilibrium of interest:

$$
\lambda_{1}=0, \quad \lambda_{2,3}= \pm i \sqrt{-2 \alpha} .
$$

(iv) The smooth function $C \in C^{\infty}\left(R^{3}, R\right)$ given by

$$
C(x, y, z)=x^{2}+2 x z-2 \alpha y^{2}+z^{2}
$$

has the following properties.

(i) It is a constant of motion for the dynamics (20)-(21).

(ii) $\nabla C\left(e^{M}\right)=0$.

(iii) $\left.\nabla^{2} C\left(e^{M}\right)\right|_{W \times W}>0$,

where

$$
W:=\operatorname{ker} d H\left(e^{M}\right)=\operatorname{span}_{R}\left(\left[\begin{array}{l}
1 \\
0 \\
1
\end{array}\right]\right) .
$$


The assertion follows via Moser's theorem for zero eigenvalues.

\section{Conclusion}

The paper presents Hamilton-Poisson realizations of a dynamical system which represents a smooth linear version of Chua system [1]. As in many other examples (Toda Lattice [6], Lü system [7], Kowalevski top dynamics [8], and battery model [9]) the Poisson geometry offers us a different point of view, unlike other old approaches, and specific tools to study the dynamics. Due to its chaotic behavior, finding its exact solutions is an open problem. This problem can be solved for the parameter values for which it admits a Hamilton-Poisson realization. In this case, the solution is given as an intersection between two surfaces: $H=$ const. and $C=$ const. In addition, we can apply energy-Casimir method to study the stability of the equilibria and Moser's theorem to find the period orbits around this equilibria. Unfortunately, these methods cannot be used for any parameters values but only for some specific ones.

\section{Acknowledgments}

The author would like to thank the referees very much for their valuable comments and suggestions. This paper was supported by the Development and Support of Multidisciplinary Postdoctoral Programmes in Major Technical Areas of National Strategy for Research-DevelopmentInnovation project $4 \mathrm{D}$-POSTDOC, Contract no. POSDRU/89/1.5/S/52603, cofunded by the European Social Fund through Sectorial Operational Programme Human Resources Development 2007-2013.

\section{References}

[1] L. O. Chua, "Nonlinear circuits," IEEE Transactions on Circuits and Systems, vol. 31, no. 1, pp. 69-87, 1984, Centennial special issue.

[2] J.-M. Ginoux and B. Rossetto, "Differential geometry and mechanics: applications to chaotic dynamical systems," International Journal of Bifurcation and Chaos in Applied Sciences and Engineering, vol. 16, no. 4, pp. 887-910, 2006.

[3] M. W. Hirsch, S. Smale, and R. L. Devaney, Differential Equations, Dynamical Systems, and an Introduction to Chaos, Elsevier, Academic Press, New York, NY, USA, 2003.

[4] B. Hernández-Bermejo and V. Fairén, "Simple evaluation of Casimir invariants in finite-dimensional Poisson systems," Physics Letters A, vol. 241, no. 3, pp. 148-154, 1998.

[5] P. Birtea, M. Puta, and R. M. Tudoran, "Periodic orbits in the case of a zero eigenvalue," Comptes Rendus Mathématique. Académie des Sciences. Paris, vol. 344, no. 12, pp. 779-784, 2007.

[6] P. A. Damianou, "Multiple Hamiltonian structures for Toda systems of type A-B-C," Regular \& Chaotic Dynamics, vol. 5, no. 1, pp. 17-32, 2000.

[7] C. Pop, C. Petrişor, and D. Bălă, "Hamilton-Poisson realizations for the Lü system," Mathematical Problems in Engineering, vol. 2011, Article ID 842325, 13 pages, 2011.

[8] A. Aron, P. Birtea, M. Puta, P. Şuşoi, and R. Tudoran, "Stability, periodic solutions and numerical integration in the Kowalevski top dynamics," International Journal of Geometric Methods in Modern Physics, vol. 3, no. 7, pp. 1323-1330, 2006.

[9] A. Aron, G. Girban, and S. Kilyeni, "A geometric approach of a battery mathematical model for on-line energy monitoring," in Proceedings of the International Conference on Computer as a Tool (EUROCON '11), pp. 1-4, Lisbon, Portugal, April 2011. 


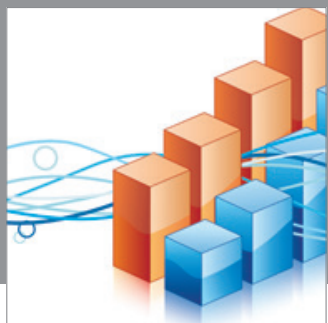

Advances in

Operations Research

mansans

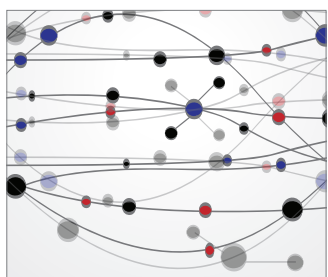

The Scientific World Journal
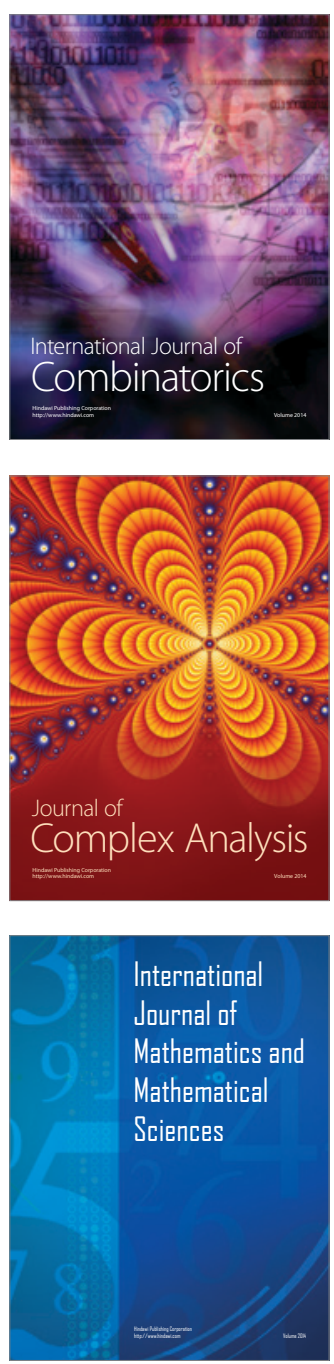
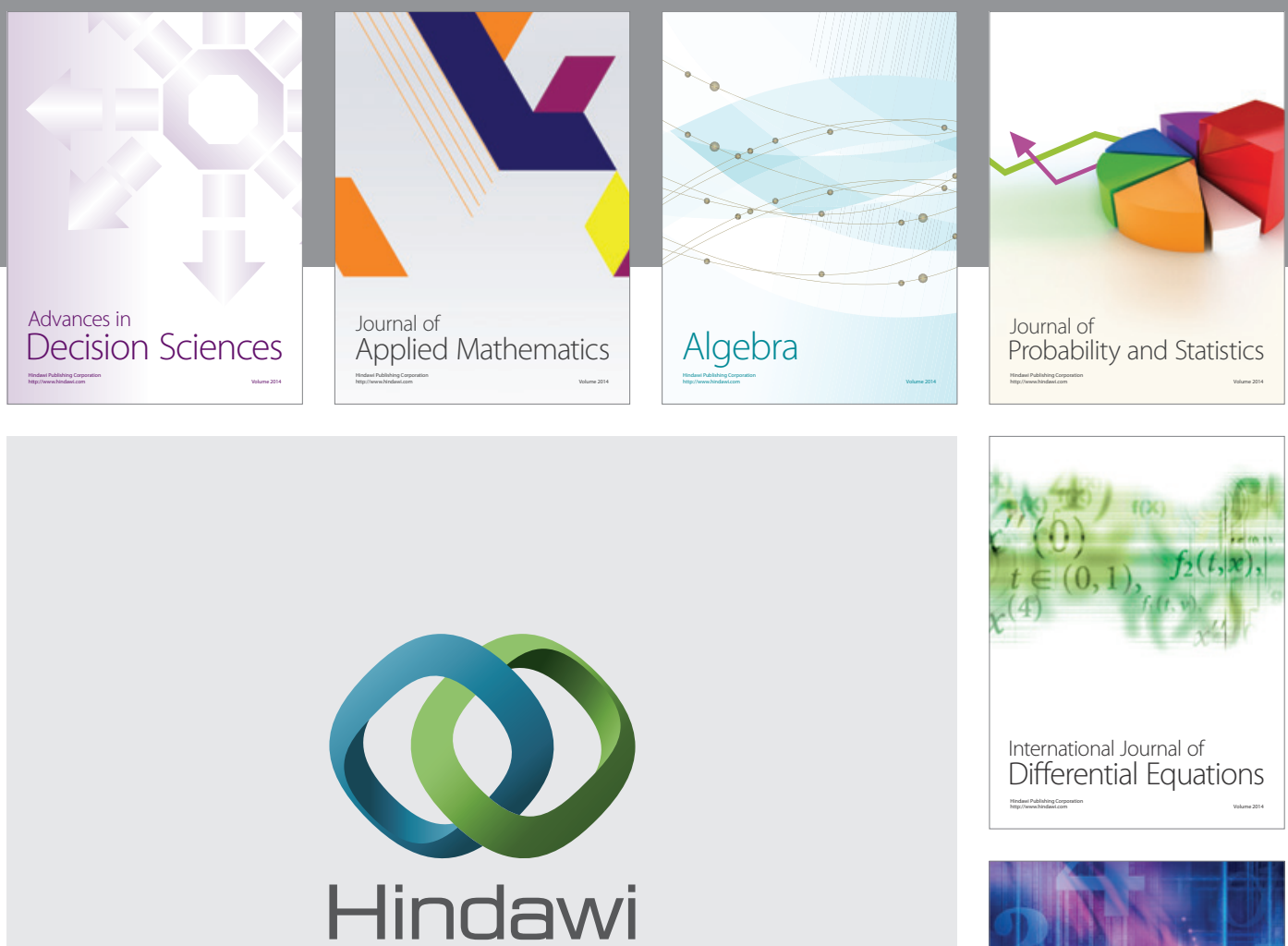

Submit your manuscripts at http://www.hindawi.com
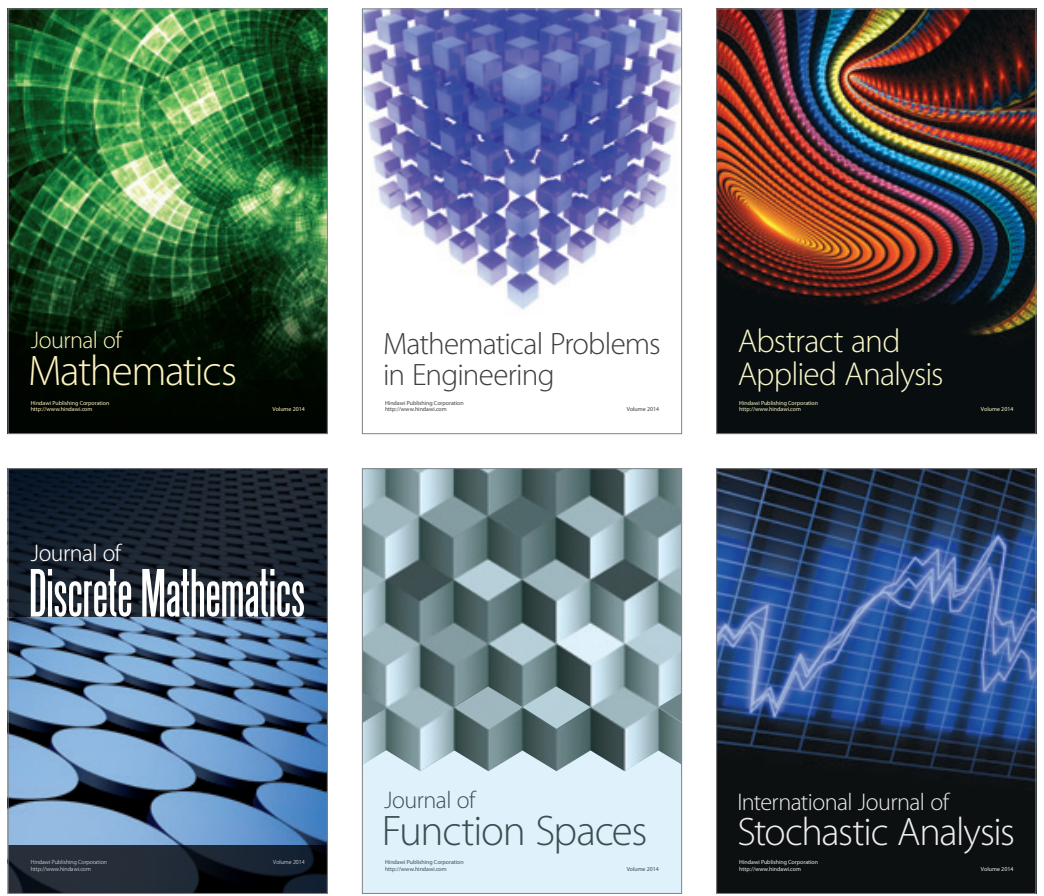

Journal of

Function Spaces

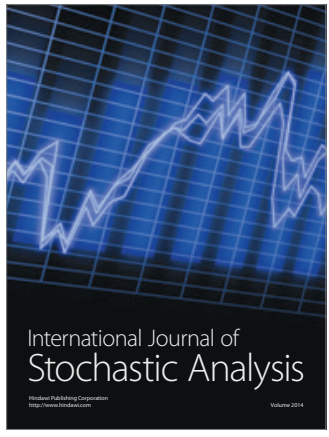

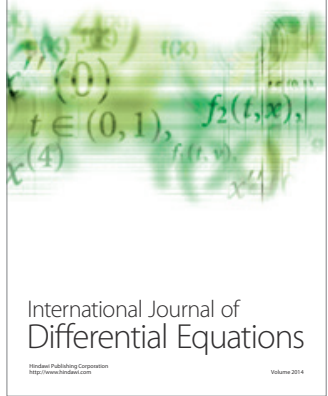
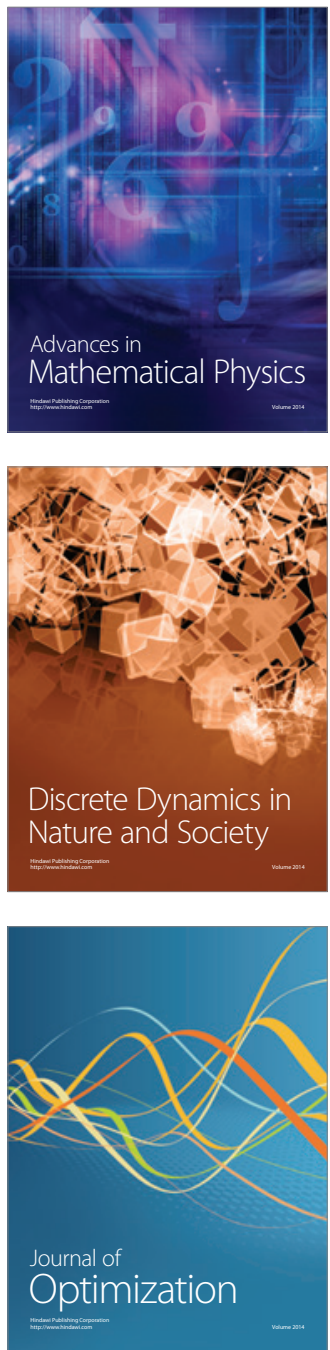